\title{
Versuch einer neuen Pleistozän-Gliederung
}

\author{
Von Julius Hesemann, Krefeld \\ Mit 4 Tabellen
}

\begin{abstract}
$\mathrm{Zus}$ ammenfassung. Die Fülle altbekannter und neuerdings gefundener Bildungen sowie physikalischer Messungen des Pleistozäns legt ein intergredierendes Gliederungsschema zur wahlweisen Einordnung der Fakten nahe. Es basiert auf einer durch die Kalt- und Warmzeiten repräsentierten Zyklik und ist auf Mitteleuropa bezogen. Die Einwirkung von mindestens 25 Zyklen auf die Tier- und Pflanzenwelt wird diskutiert.

$\mathrm{Summary}$. The abundance of old an new sediments and also physical measurements of Pleistocene suggest an integrating schema of organization to classify the facts by choice. This schema is based on a cycle reprented by the cold and warm periods and refers to Mid-Europe. The influence of at least twenty-five cycles on fauna and flora is discussed.
\end{abstract}

Die Gliederung des Pleistozäns erscheint in den derzeitigen Aufstellungen unbefriedigend. Unausgefüllt bleibt vor allem die Lücke zwischen der ältesten Kaltzeit und dem Pliozän, deren Schließung durch ungewöhnliche Verlängerungen voraufgegangener Kaltzeiten nicht überzeugen kann. Aber auch das klassische, inzwischen auf sechs (Weichsel-, Saale-, Elster-, Menap-, Eburon-, Brüggen-) Kalt- und Warmzeiten erweiterte System wird durch die Auffindung zwischenzeitlicher Boden- und Vegetationsprofile, neuer Terrassen und glaziärer Sequenzen fraglich. Das Unbehagen wird durch die Aufteilung von Kalt- und Warmzeiten auf Grund von Wärmeschwankungen von ungewisser Dauer (Großes Interglazial und Interglazial, Großes Interstadial und Interstadial, Schwankung und Horizont usw.) nicht beseitigt, sondern nur verlängert. Dabei ist der Zug vom Oligo- zum Polyglazialismus unverkennbar, nur vermißt man eine einleuchtende und die neuen Beobachtungen „intergrierende“ Pleistozänstratigraphie.

In bisher noch nicht gekannter Vielseitigkeit stehen $\mathrm{n}$ e u e F a k t e n zur Verfügung: glaziäre Sequenzen, Böden und Diskordanzen, Faunen und Floren, Geschiebe-, Geröllund Schwermineral-Gesellschaften, physikalische Altersdaten und Paläotemperaturen. Ihre Auswertung und Zusammenfügung wird beeinträchtigt durch

1. die fehlende Unterscheidung großer und kleiner Wärmeschwankungen (um eine Potenz, entweder wenige 100 bis 1000 oder \pm 10000 Jahre),

2. die Ignorierung jener Kalt- und Warmzeiten, welche keine sichtbaren Äquivalente in Moränen, Terrassen und Hochstränden hinterlassen, weil sie in der Ausdehnung, Eintiefung oder in Hochstränden hinter ihren Vorläufern zurückblieben und sich (wie z. B. in über $40 \mathrm{~m}$ hohen Terrassenstufen) nicht abheben,

3. die Unvereinbarkeit der Annahme einer Repräsentation des Pleistozäns durch lediglich etwa 10 Eiszeiten (unter Berücksichtigung von Weichsel I und II, Saale I und II usw.) mit der viel größeren Zahl von Terrassen und Zeitabschnitten ozeanischer Minimal- und Maximal-Temperaturen,

4. das Fehlen eines zeitlichen, übergeordneten Maßstabes in Form einer Periodizität, welche die Wärmeschwankungen reguliert und die Akkumulation wie die Erosion in einen immer wiederkehrenden Ablauf zwingt, den Fauna und Flora ebenfalls widerspiegeln (Zyklik).

Ich halte einen Versuch, alle bekannten Alters- und Paläotemperatur-Messungen sowie alle bekannten pleistozänen Bildungen einschließlich der Fauna und Flora miteinander in Beziehung zu setzen und in ein zeitliches Schema einzuordnen, trotz aller Lückenhaftigkeit als Zwischenergebnis schon jetzt für klärend und anregend. Das pleistozäne Geschehen 
entspricht zweifellos einer Periodik. Sie tritt uns in verschiedener Ausbildung entgegen: in der Terrassenbildung (Schotter-Hochflutlehm-Hochflutlehm-Schotter), in den Bodenprofilen (Tundragley-Parabraunerde-Braunerde-Tundragley), in der Waldentwicklung (Kiefern/Birken - Zeit - Eichenmischwald - Birken/Kiefern - Zeit), in der Faunenfolge (Lößschnecken/arktische Wirbeltiere-Steppen- und Waldtiere-Lößfauna) oder in den glaziären Sequenzen (Löß-Vorschüttsande-Moräne-Nachschüttsande-Löß).

Sucht man die Periodik als Phasenfolge zu klassifizieren, so entspricht sie einer Zyklik oder Cyclothemen. Nimmt man als eine der Voraussetzungen zur Bildung von Sedimentations-Zyklen eustatische Meeresspiegelschwankungen an, wie sie dem Gletscherhaushalt, Terrassen und Hochstränden entsprechen, dann hat man den Maßstab für den Ablauf und die Dauer der pleistozänen Kalt- und Warmzeiten gefunden (Tabellen 2 und 3). Setzt man die Cyclotheme in Beziehung zu den ozeanischen Wärmeschwankungen (EMILIANI 1966, Mesolella u. a. 1969, für Zentraleuropa ŠEgota 1967), zu den Ergebnissen der $\mathrm{K} /$ Ar-Methode (Frechen \& Lippolt 1965) und zu den Berechnungen (Tab. 1) von SoerGEL (1937) und ZEUner (1939), so ergibt sich, soweit überhaupt Bestimmungen vorgenommen wurden, eine gute Übereinstimmung. Differenzen erklären sich aus dem Bildungszeitraum von Terrassen und glaziären Bildungen. Manche Terrassen waren für Zeuner und Soergel nicht erkennbar, so daß die Zuordnung der beobachteten Terrassen oder Kaltzeiten zu den Eiszeiten eine zu frühe Datierung ergeben mußte (Tab. 1). Die Namensgebung ist jedoch eine Sache der Konvention und hat mit der Einordnung der Kalt- und Warmzeiten in den jeweiligen Zyklus nichts zu tun. Wichtig ist aber die Dauer eines Zyklus von rund 40000 Jahren, eine Zahl, welche sich aus den Altersmessungen ergibt und mit der Dauer der Cyclotheme des flözführenden Oberkarbons (JESsEN 1961) übereinstimmt.

Tabelle 1

Das Alter der Kaltzeiten nach verschiedenen Methoden und ihre Benennung ach Soergel (1937).

\begin{tabular}{|c|c|c|c|c|c|}
\hline \multirow[b]{2}{*}{ Zyklität } & \multicolumn{5}{|c|}{ Alter in 1000 Jahren nach } \\
\hline & $\begin{array}{c}\text { Kalium/ } \\
\text { Argon- } \\
\text { Methode } \\
\text { FrECHEN \& } \\
\text { LipPOLD 1965 }\end{array}$ & $\begin{array}{c}\text { Ozeanische Minimal- } \\
\text { temperaturen } \\
\text { (EMILIANI 1966) }\end{array}$ & $\begin{array}{c}\text { ZeUner } \\
1939\end{array}$ & $\begin{array}{c}\text { SoERGEL } \\
1937\end{array}$ & $\begin{array}{c}\text { Eiszeit } \\
\text { (SoERGEL 1937) }\end{array}$ \\
\hline 20 & 20 & 20 & 25 & 20 & Würm 3 \\
\hline 60 & 60 & 60 & 72 & 70 & Würm 2 \\
\hline 100 & 116 & 110 & 115 & 110 & Würm 1 (Warthe) \\
\hline 140 & 140 & 150 & 145 & 140 & Riß 3 \\
\hline 180 & - & - & 187 & 185 & $\operatorname{Riß} 2$ \\
\hline 220 & 220 & 235 & 230 & 230 & Riß 1 \\
\hline 260 & - & 270 & - & - & - \\
\hline 300 & 300 & - & 305 & 305 & Präriß \\
\hline 340 & 340 & - & - & - & - \\
\hline 380 & - & - & 395 & 395 & Mindel 3 \\
\hline 420 & 420 & 434 (ŠEGOTA 1967) & 435 & 430 & Mindel 2 \\
\hline 460 & - & 464 (Š EGOTA) & 476 & 475 & Mindel 1 \\
\hline 500 & - & - & - & - & - \\
\hline 540 & - & - & 550 & 545 & Günz 2 \\
\hline 580 & 570 & - & 590 & 590 & Günz 1 \\
\hline
\end{tabular}


Es hat aber, wie z. B. in der Weichsel-Eiszeit, etliche dicht aufeinander folgende Wärmeschwankungen gegeben, welche nicht durch einen Temperaturanstieg wie bei Interglazialen, sondern durch ihre Häufung und Gesamtdauer eine (wenn auch in unseren Breiten abgeschwächte Zyklik und) Teilung einer Eiszeit zum Ausdruck bringen.

Für die rückwärtige Verfolgung der Kaltzeiten sind hier drei Kriterien benutzt worden: Rhein-Terrassen, K/Ar-Daten und das Villafranc mit seinen Lößprofilen (BRUNNACKer 1967). Terrassen lassen sich am Mittelrhein (Quitzow 1962) und an der Ruhr bis zu den Oberen Höhen (Homberg-, Mettmann)-Terrassen ${ }^{1}$ ) verfolgen (Tab. 3). Stellt man die teilweise (bis $60 \mathrm{~m}$ ) großen Abstände in Rechnung, lassen sich diese Terrassen nach Zyklen bis zum Abschnitt der Eintiefung der Terrassentreppen in die vorhergegangene Trogregion etwa um die Zeit von 0,8 Mio Jahren aufteilen. Veranschlagt man für die Zeit der Eintiefung der Trogregion zum Hochtal um $50 \mathrm{~m}$ die Dauer von vier Cyclothemen, so gelangt man bis zur Zeitmarke 0,94 Mio Jahre und damit nahe an das älteste physikalisch datierte Glazial (in Kalifornien) vor 0,98 Mio Jahren (Tab. 3).

Auf diese Weise ergibt sich ein mindestens 25 Eiszeiten umfassender Polyglazialismus. Es bleibt nur übrig, diese theoretisch gefolgerten Kaltzeiten auch durch Sedimente zu belegen. Einen Weg dazu hat BRUNNACKER mit seinen Schülern durch die planmäßige B est a nds a uf n a h m e weit zurückreichender Quartärprofile am Mittelrhein mit Kärlich (Brunnacker, Streit \& Schirmer 1969) und am Niederrhein mit Brüggen (Boenigk 1969) und Frechen (Kowalzik 1970) beschritten. Dabei sind mehrere Löß- und Terassensysteme oberhalb der Hauptterrasse aufgefunden worden, welche die große Lücke zwischen Villafranc und Tegelen ausfüllen können und über ein einziges „Prätiglium“ hinausgehen dürften. Versucht man das Villafranc von St. Vallier als B a s is des Ple is to$\mathrm{z} \ddot{\mathrm{a} n \mathrm{~s}}$ mit seinen drei Bodenprofilen und dem liegenden Periglazialschotter von Chambaran an die älteren Rheinterrassen anzuschließen, gelangt man für das hier fixierte älteste Glazial ebenfalls zu einem Alter von 0,9 Mio Jahren. Eine Diskordanz trennt das Villafranc von St. Vallier von ariden Liegendschottern, welche wohl ohne Bedenken dem Pliozän zugeordnet werden können (Tab. 3). Die Basis des Villafranc ist altersmäßig mit rund 1,6 Mio Jahren bestimmt. Es bleibt also eine Lücke von 0,6 Mio Jahren von der Basis des Villafranc bis zum ersten belegten Glazial. Ein solcher Zeitraum dürfte für die Ablösung der pliopleistozänen Wirbeltier-Mischfauna von Beginn bis Ende des Villafranc nötig sein. Die ersten Terrassentreppen (Höhenterrassen) bedeuten nicht nur morphogenetisch eine Wende, sondern dürften auch unschwer eine Tieferlegung der Erosionsbasis durch das jetzt auf Norddeutschland übergreifende Inlandeis anzeigen. Fortan gab es enge Wechselwirkungen zwischen Terrassen-Einschneidung und Lage der Nordseeküste, zwischen zeitweiliger Blockierung der Nordsee und Aufschotterung.

Die Tie rwelt reagierte auf den mindestens 25 maligen Wechsel von Warm- und Kaltzeiten schließlich (von der Gegenwart her betrachtet) mit dem Verschwinden wärmeliebender Elemente (Elefant, Nashorn, Antilope, Flußpferd, Wasserbüffel, Löwe, Panther, Hyäne usw.). Lediglich die Vögel kehrten dank ihrer größeren Beweglichkeit jahreszeitlich als Zugvögel zurück. Für das Verhalten der übrigen Fauna leuchtet die Vorstellung (Kretzor 1961 nach Toepfer 1963) ein, daß in günstigen Zeiten neue Formen in Wellen oder „Fronten“ in Mitteleuropa einwanderten, sich in den Warm- (mediterrane Steppen und Wälder) und Kaltzeiten (Lößsteppen und Tundra-Gebiete) mit verschiedenem Erfolg (bei der Nichtumkehrbarkeit des Evolutionsgeschehens) anpaßten und in Warmzeiten durch neue Faunenwellen verdrängt wurden. Die einzelnen Zyklen waren indessen für die Setzung umfassender Zäsuren in der Veränderung der Tierwelt zu kurz. Abgesehen von

1) Die Zuordnung der Höhen (Mettmann-, Hösel-, Drüfel)-Terrassen zum Pliozän, der ich noch kürzlich (HESEMANN 1969) gefolgt war, ist demnach durch die Verweisung ins Altpleistozän zu berichtigen. 
Tabelle 2

Gliederungsschema des Pleistozäns

\begin{tabular}{|c|c|c|c|}
\hline $\begin{array}{c}\text { Zyklen- } \\
\text { Alter } \\
\text { in cirka } \\
1000 \text { Jahren }\end{array}$ & $\begin{array}{l}\text { Kalium-Argon-Alter } \\
\text { (FrECHEN \& Lippold } \\
1965) \\
\text { in } 10\end{array}$ & $\begin{array}{c}\text { Alter der ozeanischen } \\
\text { Maximal (Ma-) und } \\
\text { Minimal (Mi-)Temperaturen } \\
\text { (EMILIANI 1966) } \\
\text { O J a h r e n }\end{array}$ & $\begin{array}{c}\text { Terrassenhöhen am } \\
\text { Mittelrhein } \\
\text { (Qurtzow 1962) } \\
\text { in m über Rhein-Mittel- } \\
\text { wasser }\end{array}$ \\
\hline \multirow[t]{2}{*}{20} & 20 & $20 \mathrm{Mi}, 28 \mathrm{Mi}$ & 6 \\
\hline & & $48 \mathrm{Ma}, 55 \mathrm{Ma}$ & \\
\hline \multirow[t]{2}{*}{60} & 60 & $65 \mathrm{Mi}$ & 10 \\
\hline & & $80 \mathrm{Ma}$ & \\
\hline \multirow[t]{2}{*}{100} & 116 & $115 \mathrm{Mi}$ & 30 \\
\hline & & $135 \mathrm{Ma}$ & \\
\hline \multirow[t]{2}{*}{140} & 143 & $150 \mathrm{Mi}$ & \\
\hline & & $170 \mathrm{Ma}$ & \\
\hline \multirow[t]{2}{*}{180} & & $180 \mathrm{Mi}$ & 60 \\
\hline & & $210 \mathrm{Ma}$ & \\
\hline \multirow[t]{2}{*}{220} & 220 & $235 \mathrm{Mi}$ & \\
\hline & & $250 \mathrm{Ma}$ & \\
\hline \multirow[t]{2}{*}{260} & & $270 \mathrm{Mi}$ & 80 \\
\hline & & $290 \mathrm{Ma}$ (Mesolella 1969) & \\
\hline \multirow[t]{2}{*}{300} & 300 & & \\
\hline & 320 & $325 \mathrm{Ma}$ (Mesolella) & \\
\hline 340 & 340 & & 100 \\
\hline \multirow[t]{2}{*}{380} & & & 120 \\
\hline & & $395 \mathrm{Ma}$ (Mesolella) & \\
\hline 420 & 420 & $434 \mathrm{Mi}$ (ŠEGOTA 1967) & \\
\hline \multirow[t]{2}{*}{460} & . & $464 \mathrm{Mi}$ (ŠEGOTA) & 140 \\
\hline & & $485 \mathrm{Ma}$ (Mesolella) & \\
\hline \multirow[t]{2}{*}{500} & & & 170 \\
\hline & 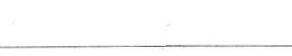 & , & \\
\hline \multicolumn{4}{|l|}{540} \\
\hline 580 & 570 & senta & 200 \\
\hline 620 & & & \\
\hline
\end{tabular}


seit 0,6 Mio Jahren $\mathrm{nach} \mathrm{Zyklen}$

\begin{tabular}{|c|c|c|c|}
\hline $\begin{array}{c}\text { Rheinterrassen, () vermutlich } \\
\text { in der gleichen Terrassenstufe } \\
\text { enthalten }\end{array}$ & $\begin{array}{l}\text { Kalt- u. } \\
\text { Warmzeiten, } \\
\text { () theoretısch }\end{array}$ & $\begin{array}{l}\text { Mögliche } \\
\text { Einordnung }\end{array}$ & $\begin{array}{c}\text { (Eu) Homininen } \\
\text { (Hebeler \& Kurth 1966) }\end{array}$ \\
\hline \multirow[t]{2}{*}{ Untere Niederterrasse } & Kaltzeit 1 & Weichsel 2 & Homo sapiens sapiens \\
\hline & Warmzeit & $\begin{array}{l}\text { Moershoofd / } \\
\text { Poperinge-Torfe }\end{array}$ & \\
\hline \multirow[t]{2}{*}{ Obere Niederterrasse } & Kaltzeit 2 & Weichsei 1 & H. sapiens neanderthalensis \\
\hline & Warmzeit & Eem & H. sapiens praeneanderthalensis \\
\hline \multirow[t]{2}{*}{ Unt.Mittelterr.,Krefeld,Mittelterr. } & Kaltzeit 3 & Warthe & \\
\hline & Warmzeit & Ohe ? & \\
\hline \multirow[t]{2}{*}{ Untere Mittelterr. (Talweg-Terr.) } & Kaltzeit 4 & Drenthe & \\
\hline & Warmzeit & Holstein & H. sapiens praesapiens \\
\hline \multirow[t]{2}{*}{ Mittl. Mittelterr. (Rinnenschotter) } & Kaltzeit 5 & 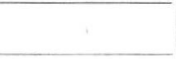 & \multirow{6}{*}{ Homo erectus heidelbergensis } \\
\hline & Warmzeit & & \\
\hline \multirow[t]{2}{*}{ (Oberer Mittl. Mittelterrasse) } & Kaltzeit 6 & & \\
\hline & Warmzeit & & \\
\hline \multirow[t]{2}{*}{ Obere Mittelterrasse } & Kaltzeit 7 & Elster & \\
\hline & Warmzeit & & \\
\hline \multirow[t]{2}{*}{ Oberste Mittelterrasse } & Kaltzeit 8 & & \multirow{6}{*}{ Homo erectus } \\
\hline & Warmzeit & & \\
\hline \multirow[t]{2}{*}{ Untere jüng. Hauptterrasse } & Kaltzeit 9 & & \\
\hline & Warmzeit & Cromer 2 & \\
\hline \multirow[t]{2}{*}{ Obere jüng. Hauptterrasse } & Kaltzeit 10 & & \\
\hline & Warmzeit & Cromer 1 & \\
\hline \multirow[t]{2}{*}{ (Ob. jüng. Hauptterrasse) } & Kaltzeit 11 & Menap & \\
\hline & Warmzeit & Waal & \\
\hline \multirow[t]{2}{*}{ Mittlere Hauptterrasse } & Kaltzeit 12 & Eburon & \\
\hline & Warmzeit & Tegelen 2 & \\
\hline \multirow[t]{2}{*}{ Obere Hauptterrasse } & Kaltzeit 13 & & \\
\hline & Warmzeit & Tegelen 1 & \\
\hline \multirow[t]{2}{*}{ (Obere Hauptterrasse) } & Kaltzeit 14 & Brüggen & \\
\hline & Warmzeit & & \\
\hline \multirow[t]{2}{*}{ Untere (Drüfel) Höhenterrasse } & Kaltzeit 15 & & \\
\hline & Warmzeit & & \\
\hline Untere (Hösel) Höhenterrasse & Kaltzeit 16 & & \\
\hline
\end{tabular}


Tabelle 3

Gliederungs chema des Pleistozäns

vom Pliozän bis vor 0,6 Mio Jahren nach Zyklen

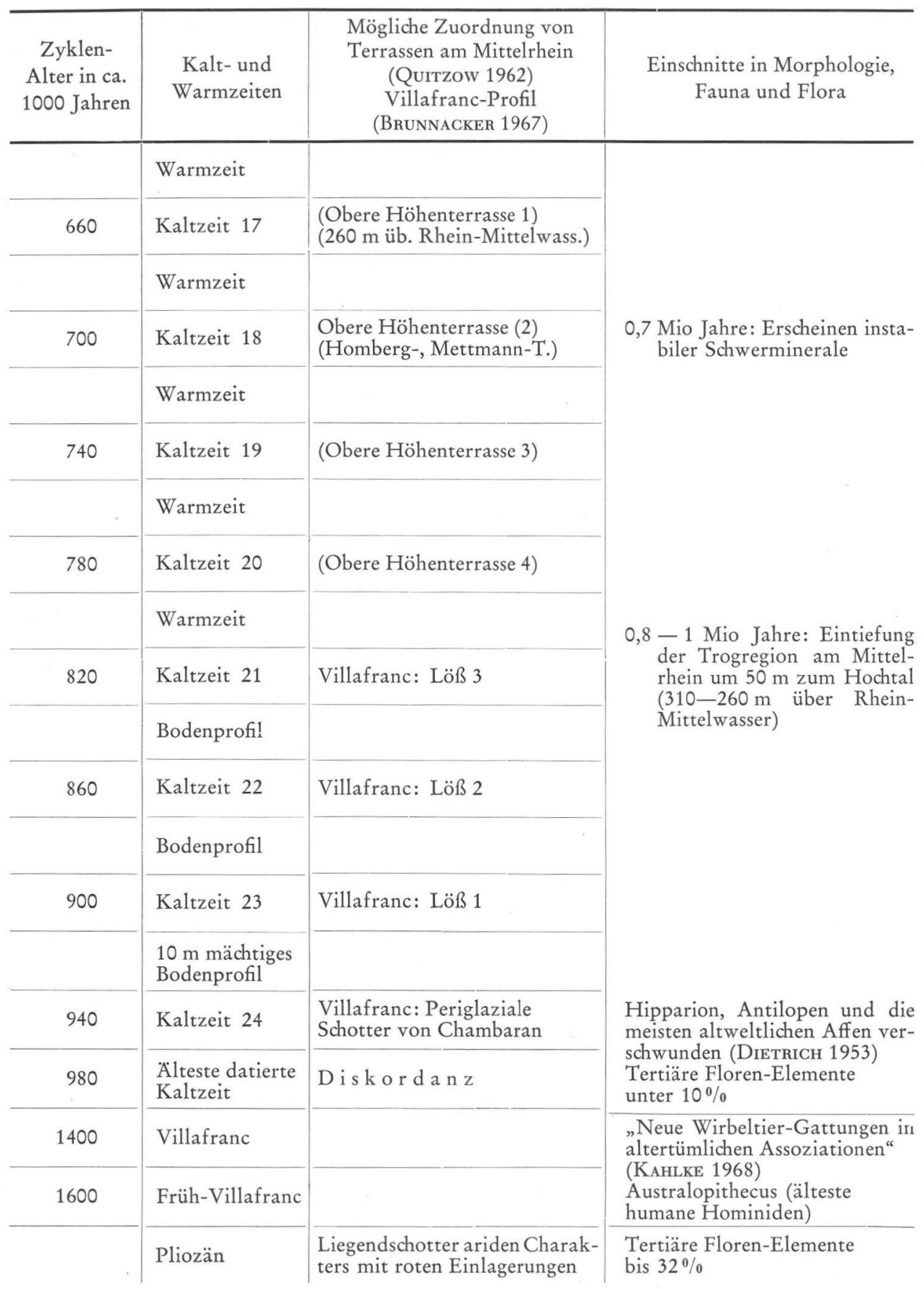


Dauerformen (z. B. Dicerorbinus etruscus, D. kirchbergensis) lösen (besonders unter den Kleinsäugern, ToEPfer 1963) einzelne Gattungen einander ab oder (bei den Großsäugern) Stammreihen entwickeln sich. Die Lebensdauer von Arten und Gattungen liegt dabei vielfach zwischen 0,1-0,3 Mio Jahren.

Man hat deshalb die Glieder der Elefanten- oder Pferde-Stammreihen als Maßstäbe für die Pleistozän-Gliederung genommen und so Alt-, (vorher auch noch Ältest)-, Mittelund Jungpleistozän unterschieden, ohne aber die Ungleichheit der Zeitabschnitte zu betonen. Von den (im Verhältnis zur theoretischen Vielzahl) überlieferten wenigen Faunen zeichnen sich jedoch einige dadurch aus, daß besonders viele alte Formen erloschen sind und besonders viele neue Formen erscheinen. Sechsmal fällt ein solcher Wechsel auf:

1. Am Ende des Früh-Villafranc, vor etwa 1,4 Mio Jahren.

Nicht mehr vorhanden: Zyglodon borsoni, Ursus etruscus,

neu: Desmana vebringi, Mimomys pliocaenicus, Sus arvernensis, Leptobos elatus, Hystrix refossa.

2. Am Ende des Villafranc, vor 1 Mio Jahren.

Nicht mehr vorhanden: Soriculus, Prospalax,

neu: Equus stenonis, Allobippus robustus, Dicerorbinus etruscus, Dic. kirchbergensis, Archidiskon meridionalis, Elephas planifrons, Crocuta perrieri.

3. Am Ende der Tegelen-Warmzeit, vor 0,5 Mio Jahren.

Nicht mehr vorhanden: Leptobos elatus, Macaca florentina, Talpa präglacialis, Ursus etruscus,

neu: Desmana tegelensis, Sus strocci, Desmana tegelensis, Hystrix etrusca, Dolomys milleri, Trogontherium cuvieri.

4. Am Ende der Cromer-Warmzeit, vor 0,32 Mio Jahren.

Nicht mehr vorhanden: Equus stenonis, Archidiskon meridionalis, Trogontherium, neu: Ursus spelaeus, Sorex, Discrostonyx Microtus, Desmana moschata mosbachensis, Ovibos, Bison schoetensacki, Caprea, Megaloceras, Orthogonoceras, Capreolus, Rangifer, Palaeoxolon antiquus, Mammontheus trogontherium, Hippopotamos, Machairodus, Equus süssenbornensis, E. mosbachensis, Sus scrofa.

(Ersatz von Leptobos durch Bos und Bison, der "Quaggas“ durch caballische Pferde und von Archidiskon meridionalis durch Mammontheus trogontheri, Dietrich 1953).

5. Am Ende der Elster-Kaltzeit vor 0,18 Mio Jahren.

Nicht mehr vorhanden: Archidiskon meridionalis, Hystrix refossa, Orthogonoceras, Dolichodoryceras, Hippopotamus, Equus mosbachensis, neu: Ursus arctos, Gulo.

6. Am Ende der Eem-Warmzeit, vor etwa 0,08 Mio Jahren.

Nicht mehr vorhanden: Megaloceras, Bison priscus, Equus caballus, Dicerorbinus etruscus, Dic. kirchbergensis, Bos primigenius, Buffelus, Saiga tatarica, Palaeoloxodon antiquus,

neu: Mammontheus primigenius, Equus caballus, E. germanicus, Coelodonta antiquitatis, Buffelus, Saiga tatarica.

Aus der allmählich dichteren Aufeinanderfolge (wohl kein Trugschluß wegen der zunächst dürftigeren Belege) der „Faunenwellen“ ergibt sich für das Altpleistozän ein langsameres, für das Jungpleistozän ein sich steigerndes Tempo der Evolution. Aber so wenig der vorgeschichtliche Mensch durch die Jagd die Wirbeltierwelt dezimiert hat (SoergeL 1922), so gewiß ist dem Homo sapiens sapiens die derzeitige Verarmung der Wirbeltierwelt zur Last zu legen. 
Auch in der Entwicklung der Hominiden scheinen Haupteinschnitte an der Grenze Plio/Pleistozän und um die Cromer-Warmzeit zu liegen. Die Plio-Pleistozän-Grenze bedeutet das Übergangsfeld von der „subhumanen Phase“ der Hominiden über das Tier hinaus zur humanen Phase in Gestalt der Australopithecinen, welche als „pygmoide kleinhirnige Aufrechtgänger mit typischen Standfüßen und grundsätzlich menschlichem Gebiß“ (HEBERER) sich schon mancher Werkzeuge und Geräte bedienten. Ihnen folgten die Frühmenschen mit (Pithecanthropus) Homo erectus (heidelbergensis als ältester Fund in der Elster-Kaltzeit) und diesen verhältnismäßig rasch aufeinander die Alt- (Homo sapiens neanderthalensis) und Jetztmenschen (Homo sapiens praesapiens $u$. H. sapiens sapiens) in der letzten Warm- und Kaltzeit (Heberer \& Kurth 1966). Also anscheinend wie bei den Wirbeltieren allgemein eine beschleunigte Evolution.

Bei den marinen Mollusken zeichnen sich der Einbruch der Kaltzeiten und der seitdem waltende Zyklus von Kalt-Warmzeiten mit der Mischung und Ablösung lusitanischer durch boreale Formen ab' (vgl. Verzeichnis der Schnecken und Muscheln bei van DER KLERK \& Florschütz 1953). Mit dem Aufhören besonders vieler alter und dem Erscheinen neuer Arten hebt sich die Cromer-Warmzeit als Wende ab, während Holstein-See und Eem-Meer sich durch das Ausscheiden weniger Formen (Corbula fluminalis, Vivipara diluviana, Valvata naticina) voneinander unterscheiden.

Eine gegensätzliche Entwicklung lehrt die Geschichte der pleistozänen Vegetation (soweit bei der Verschiedenheit pflanzlicher und tierischer Baupläne überhaupt ein Vergleich möglich ist). Nachdem bereits im Unteroligozän Mitteleuropas die ersten „arktotertiären“ Floren-Elemente erschienen waren (AHRENS u. a. 1968), kommen sie in der Folgezeit in einem oftmaligen Wechsel zum Ausdruck. Im Pliozän aber kam es zu erheblichen Schwankungen in der Beteiligung typischer Tertiärformen (Tab. 4). Noch bleiben einschneidende Absenkungen der Temperatur aus, aber im Reuver-Abschnitt partizipieren als Vorboten einer erheblichen Abkühlung Betula und Pinus silvestris zeitweilig mit 50\% an der $\mathrm{Zu}-$ sammensetzung der Baumpollen, während die tertiären Formen auf den bis dahin noch nicht erreichten Tiefstand von wenig über $10 \%$ zurückgehen (Tab. 4). Diese Tendenz setzt sich fort, bis in der Cromer-Warmzeit der Umbau der tertiären Waldvegetation in die pleistozäne warmzeitliche Waldvegetation vollzogen ist (FrENzEL 1968). Von nun an läuft in jeder Warmzeit eine schnelle Folge scharf getrennter Waldtypen (Birke/Kiefer, Kiefer/ Birke/Fichte/Ulme, Eichenmischwald, Hainbuchen/Tanne oder Carpinus/Fichte, Kiefer/ Fichte/Birke/Kiefer) ab, nur in der Holstein-Warmzeit durch eine starke Vorherrschaft von Nadelhölzern verschleiert.

Es bedurfte mithin der Einwirkung von Kalt- und Warmzeiten über 1,3 Mio Jahre hindurch, um die tertiären Baumpollen in Mitteleuropa auszuschalten. Aber während die Wirbeltiere auf diese periodischen Impulse einer veränderten Umwelt mit der Schöpfung ganzer Ketten von Stammesreihen reagierten, verhielten sich die Pflanzensippen physiologisch konservativ und wenig variabel. Abgesehen von der fortschreitenden Verarmung an tertiären Elementen konsolidierte sich die pleistozäne Vegetation in der Bildung zweier $\mathrm{S}$ t a nd a rdt y p e n, die sich als Siedlungsgemeinschaften von Kältesteppen mit nur (für Mitteleuropa) kurzzeitig und schwach ausgeprägter Tundra und als Eichenmischwald mit einer Nadelwald-Phase am Anfang und am Ende präsentierten. Beide besetzten immer wieder je Kalt- oder Warmzeit die gleichen Areale (Frenzel 1968), wobei sie sich aus den Refugien des Mittelmeerraumes oder den nordeuropäischen Tundren ergänzten. Dabei erwiesen sich Sumpf- und Wasserpflanzen als besonders konservativ. Die großen Einschnitte in der Entwicklung während des Pleistozäns liegen — wie bei den Wirbeltieren - im Früh-Abschnitt des Villafranc und in der Cromer-Warmzeit, so daß eine Wechselwirkung naheliegt. Wie die Evolution der Tierwelt verlangt auch die Vegetationsgeschichte des Pleistozäns die 1,3 Mio Jahre als Frist für die Reifung. Diese große und entscheidende Zeit 
Tabelle 4

Der Anteil typischer Baumpollen an der Vegetation von Oberpliozän und Pleistozän (im wesentlichen nach ZAGwiJN a us FrenzeL 1968 ) in NW-Europa

\begin{tabular}{|c|c|c|c|c|c|}
\hline \multirow{3}{*}{ Zeit-Abschnitte } & \multirow{3}{*}{$\begin{array}{c}\text { Typische } \\
\text { Tertiärformen }\end{array}$} & \multicolumn{4}{|c|}{ Zeitweiliger maximaler Anteil an } \\
\hline & & $\begin{array}{l}\text { Eichenmisch- } \\
\text { wald }\end{array}$ & $\begin{array}{c}\text { Pinus } \\
\text { silvestris }\end{array}$ & Betula & Picea \\
\hline & & \multicolumn{4}{|c|}{ in Prozenten } \\
\hline Eem-Warmzeit & 0 & 80 & 90 & 90 & 12 \\
\hline Holstein-Warmzeit & 0 & 10 & 70 & 10 & 20 \\
\hline Cromer-Warmzeit & $1-0$ & 80 & 85 & 70 & 15 \\
\hline Waal-Warmzeit & 4 & 45 & & & \\
\hline Tegelen-Warmzeit & 8 & 12 & 80 & 6 & 55 \\
\hline Prätegelen & 2 & 3 & & & \\
\hline Reuver (Asti) & $12-38$ & 40 & 50 & 35 & 4 \\
\hline Brunssumian C & $20-70$ & & & & \\
\hline ” $\quad \mathrm{B}$ & $20-72$ & & & & \\
\hline (Piacentin) $A$ & $30-70$ & & & & \\
\hline Susterian (Pont) & $20-30$ & & & & \\
\hline
\end{tabular}

zwischen Pliozän und Cromer-Warmzeit ist geologisch und biologisch (Gleichsetzung von Villafranc und Tegelen, Annahme nur weniger Kaltzeiten vor der Tegelen-Warmzeit) nicht genügend gewürdigt. In den großen Einschnitten der Lebewelt vor 1,6 und 0,3 Mio Jahren darf man außer einer Verzahnung biologischer Zeitenwenden (DIETRICH 1953) auch eine, im großen gesehen ständige, noch nicht beendete Abkühlung seit dem Pliozän erblicken. Das wäre auch insofern nicht unwahrscheinlich, als die Gegenwart erst beim 25. Zyklus der Kaltzeiten angelangt ist und (wie im Permokarbon) ein Vielfaches noch bevorsteht.

Úberblickt man die Entwicklung von Fauna und Flora im Quartär, die Dokumentierung von Kalt- und Warmzeiten durch Moränen, Lösse, Terrassen und Strandstände einerseits und das Ergebnis verschiedener Untersuchungsmethoden zu ihrer zeitlichen, räumlichen und klimatischen Erfassung andererseits, so ergibt sich ein im großen gerichteter und zyklisch gearteter Ablauf. Die jeweils fixierte organische und anorganische Materie entspricht dabei in ihrer Ausbildung sozusagen Variationen zum gleichen Thema, nämlich dem Arrangieren mit den durch Kalt- und Warmzeiten gebotenen Verhältnissen. Diese begrenzte Variabilität innerhalb der $\mathrm{Z}_{\mathrm{yk}} \mathrm{kik}$ ist durch den komplexen Charakter der steuernden Faktoren (Paläotopographie, sinkende Temperaturen, weichende Nordsee, extratellurische Ursachen usw.) begreiflich.

Die Bildungen des Pleistozäns müssen sich jedesmal ihren Raum und ihre Substanz größtenteils auf Kosten vorangegangener Ablagerungen selbst schaffen. Infolgedessen sind Moränen, Terrassen und Lösse wegen ihrer lockeren Beschaffenheit und exponierten Lage häufig verschwunden oder aber nach Morphologie und Zusammensetzung oft nicht von ihren Vorgängern zu unterscheiden. Die geologische Diagnose steht deshalb vor einer schwierigen Aufgabe. Sie kann sich aber der mannigfachen (von der Bodenkunde, Paläontologie, Petrographie, Morphologie, den physikalischen Alters- und TemperaturBestimmungen gebotenen) Indizien in gemeinsamer Abstimmung bedienen. Sie bleiben jedoch so lange erdgeschichtlich beziehungslos, bis für ihre Einordnung die spezifisch plei- 
stozäne zyklische Periodik zur Verfügung steht. Daß diese einer Zyklik entspricht, bedarf angesichts der kalt-warmzeitlichen, durch Fauna und Flora widergespiegelten und durch physikalische Daten zeitlich bestätigten Sedimentations-Folge keiner weiteren Beweisführung, wenn auch die letzten Ursachen dafür unbekannt sind. Auf der Grundlage der Zyklik ist die vorstehende Gliederung des Pleistozäns entworfen, eine in sich konsequente Aufteilung, zwar immer noch unvollkommen, aber mit weniger Lücken als bisher, in einer natürlichen Ordnung und mit der Möglichkeit, alte sowie neue Beobachtungen und Daten erdgeschichtlich einzuordnen.

Mag man diesen Versuch als weitere Variation der bereits vorhandenen zahlreichen Hypothesen skeptisch aufnehmen und als verfrüht ansehen, so kann er gleichwohl bei der Reformbedürftigkeit des klassischen Eiszeiten-Systems wegen seines unleugbaren Zerfalls in mehrteilige Kalt- und Warmzeiten als Lückenbüßer dienen. Das Verharren im Oligoglazialismus engt den Blick für stratigraphische Vergleiche sowie für die Geschichte der Tier- und Pflanzenwelt angesichts der vermehrten Fakten ein. Das hier über die bisherigen Aufteilungen hinausgehende $\mathrm{Schema}$ soll lediglich eine Möglichkeit $\mathrm{zur}$ wahlfreien Einordnung alter und neuer Pleistozän-Bildungen bieten und für die Beurteilung der tierischen und pflanzlichen Entwicklung mehr Stationen, vor allem im Altpleistozän, zur Verfügung stellen. Was bei dieser Gelegenheit über Evolution gesagt ist, hat mehr die Bedeutung einer Fragestellung zur Wechselwirkung von geologischem und biologischem Geschehen als einer abschließenden Feststellung, weil die Kausalzusammenhänge zwischen Evolution und Umweltänderungen noch nicht geklärt sind.

\section{Literaturhinweise}

Ahrens, H. u. a.: Zur Plio/Pleistozän-Grenze in der Deutschen Demokratischen Republik. 23. intern. geol. Congress, 10, 65-77, 5 Abb., Prag 1968.

Boenick, W.: Zur Kenntnis des Altquartärs bei Brüggen (westlicher Niederrhein). - Sonderveröff. geol. Inst. Köln, 17, 138 S., 20 Abb., 7 Tab., 3 Taf., Köln 1969.

Brunnacker, K.: Der Villafranchium-Löß bei St. Vallier. - N. Jb. Geol. Paläont., Jg. 1967, 257-267, 1 Abb., 1 Tab., Stuttgart 1967.

Brunnacker, K., Streit, R. \& Schirmer, W.: Der Aufbau des Quartär-Profils von Kärlich/Neuwieder Becken (Mittelrhein). - Mz. naturw. Arch., 8, 102-133, 8 Abb., 3 Tab., 1 Taf., Mainz 1969.

Dietrich, W. O.: Neue Funde des etruskischen Nashorns in Deutschland und die Frage der Villafranchium-Faunen. - Z. Geol., 2, 417—430, 1 Abb., Berlin 1953.

Emiliani, C.: Palaeotemperature Analysis of Core 280 and pleistocene Correlations. - Journ. of Geol., 66, 265-275, 5 Abb., 3 Tab., Chicago 1958.

Frechen, J. \& Lippolt, H. J.: Kalium-Argon-Daten zum Alter des Laacher Vulkanismus, der Rheinterrassen und der Eiszeiten. - Eiszeitalter u. Gegenwart, 16, 5-30, 8 Abb., Öhringen 1965.

Frenzel, B.: Grundzüge der pleistozänen Vegetationsgeschichte Nord-Eurasiens. - Erdwiss. Forsch., 1, 326 S., 17 Taf., 67 Abb., Wiesbaden 1968.

Heberer, G. \& Kurth, G.: Die (Eu)Hominiden vom Mittelpleistozän bis ins mittlere Jungpleistozän. - Handbuch der Urgeschichte, I, 209-233, 1 Taf., 5 Abb., 1 Tab., Bern 1966.

Hesemann, J.: Das Münsterland als pliozäne Erosionslandschaft. - N. Jb. Geol. Paläont. Mh., Jg. 1969, 530-534, 3 Tab., Stuttgart 1969.

Jessen, W.: Zur Sedimentologie des Karbons. - 4. Congr. Avancement Etudes Strat. Géol. Carbonifères, C. R., 307-322, Maastricht 1961.

KAнLKE, H.-D.: Vertebratenstratigraphie zur Plio/Pleistozän-Grenze. - Report 23. Intern. geol. Congress, Section 10, 27-39, Prag 1968.

KretzoI, M.: Stratigraphie und Chronologie. Czwartorzed europy Srodkoweji Wschodniej. Inqua, 1961, 313-329, Łódź 1964.

Mesolella, K. M., Matthews, R., Broecker, W. S. \& Thurber, D. L.: The astronomic Theory of climatic Change.: Barbados Data. - J. Geol., 77, 250-274, 13 Abb., Chicago 1969.

Quitzow, H. W.: Mittelrhein und Niederrhein. - Beitr. z. Rheinkde., 13, 27-39, 4 Abb., Koblenz 1962.

ŠEgOTA, T.: Paläotemperature Changes in the Upper and Middle Pleistocene. - Eiszeitalter u. Gegenwart, 18, 127-141, 3 Abb., O'hringen 1967. 
Soergel, W.: Die Jagd der Vorzeit. - 149 S., 28 Abb., 1 Tab., Jena 1922.

- : Die Vereisungskurve. - 87 S., 1 Taf., 3 Tab., Berlin 1937.

Toepfer, V.: Tierwelt des Eiszeitalters. - 46 Abb., 20 Taf., 2 Tab., Leipzig 1963.

VLERK, I. M. VAN DER \& FlorschüTZ, F.: The palaeontological Base of the Subdivision of the Pleistocene in the Nederlands. - Verhandl. Koninkl. nederl. Akad. van Wetensch. afd. Natuurkunde, 1, reeks, 20, Nr. 2, 58 S., 29 Tab., 4 Taf., Amsterdam 1953.

Zeuner, F. E.: Schwankungen der Sonnenstrahlung und des Klimas im Mittelmeergebiet während des Quartärs. — Geol. Rdsch., 30, 650—656, 1 Tab., Stuttgart 1939.

Manuskr. eingeg. 13. 7. 1970.

Anschrift des Verf.: Prof. Dr. J. Hesemann, 415 Krefeld, Von-Steuben-Straße 17. 